\title{
Inovações no financiamento federal à Atenção Básica
}

\author{
Brazilian federal financing innovations \\ in Basic $\mathrm{H}$ ealth
}

Clarice M elamed 1

Nilson do Rosário Costa 1

1 Departamento de Ciências Sociais, ENSP/Fiocruz.

Rua Leopoldo Bulhões 1480, 9o andar M anguinhos, 21041-210 Rio de Janeiro RJ. melamed@ensp.fiocruz.br
Abstract The current paper deals with innovations in the Brazilian federal financial framework of public basic health services. It considers the creation of new programs: Basic Attention (PAB) Community $\mathrm{H}$ ealth A gents Program (PACS) and the Family $\mathrm{H}$ ealth Program (PSF). It was built a specific framework to analyze PABs program impact over the municipalities' accounts, considering the fiscal capacity of these Federation units, their population size and geographic regions. For PACS and PSF it's presented the increasing amount of financial resources available between 1998 and 2001 by geographic regions. Considering the financial resources delivered by the Federal Government, the new programs can be analyzed as being well succeeded. The data available shows that the counties which had in the beginning less resources in order to deliver basic health services were the ones which received more financial aid. PACs and PSF programs also had had increasing federal financial resources from 1998 to 2001.

Key words $\mathrm{H}$ ealth policy financing, Basic $\mathrm{H}$ ealth Services, Redistributive policy
Resumo 0 presente artigo trata das inovações nas formas de financiamento federal à atenção básica, a partir da criação de novos programas: Piso de A tenção Básica (PAB), Programa de Agentes Comunitários (PACS) e Programa de Saúde da Família (PSF). Foi construída uma tipologia específica para a interpretação do impacto do PAB Fixo sobre as contas municipais, considerando a capacidade fiscal das unidades da Federação, seu porte populacional e região geográfica. Para o PACS e o PSF foi feita uma análise do crescimento de recursos disponibilizados ao longo do período 1998-2001, por regiões geográficas. Conclui-se que, ao menos em seu movimento inicial, a nova política é bem-sucedida: a análise dos dados de implementação do PAB demonstra que os municípios de menor capacidade instalada para a oferta de atenção ambulatorial básica no âmbito do Sistema Ú nico de Saúde foram os mais favorecidos pel os novos mecanismos de transferência propostos pelas $\mathrm{N}$ ormas $\mathrm{O}$ peracionais Básicas (N O Bs); também foram alocados crescentes volumes de recursos di recionados aos programas PACS e PSF durante o período. Palavras-chave Financiamento, Atenção Básica, Política redistributiva 


\section{Introdução}

$\mathrm{Na}$ primeira parte deste artigo, apresentamos as principais transformações ocorridas em termos da legislação em vigor e que se evidenciam no interior das contas do M inistério da Saúde, a partir de 1998, correspondentes à aplicação de novas políticas orientadas à atenção básica, particularmente o Piso de Atenção Básica (PAB), o Programa de Agentes Comunitários (PACS) e o Programa de Saúde da Família (PSF).

A implementação do PAB, definido pela Norma O peracional Básica 01/96 publicada no Diário Oficial da União de 6/11/1996, está diretamente associada ao desenvolvimento de um novo modelo assistencial. Esse modelo assistencial se efetivaria pelo deslocamento da atenção médico-hospitalar, pela meta de diminuição das internações e pela criação de programas substitutivos ou complementares de natureza ambulatorial e intersetorial, como demonstramos adiante.

Os objetivos estratégicos desse processo de inovação no modelo assistencial têm sido:

a) melhorar as condições de acesso e utilização de atenção à saúde pelo incentivo à ampliação da oferta de serviços ambulatoriais;

b) ampliar os mecanismos de referência aos serviços de apoio e diagnósticos e os de maior complexidade e custo;

c) mudar o perfil das despesas pela introdução de práticas preventivas e de serviços substitutivos de menor custo;

d) inserir a oferta de serviços no território pela responsabilização com os espaços e condições que compõem a vida cotidiana dos pacientes;

e) gerar um círculo virtuoso do gasto em saúde pela multiplicação de ações de prevenção e promoção à saúde.

As atividades desenvolvidas pelos novos programas associados parecem também definir um novo modelo de proteção social pela crescente adoção de ações orientadas a grupos de risco ou populações-alvo. Considera-se população-alvo as pessoas, habitantes, comunidades ou organizações ou outras unidades para as quais o programa está orientado ou que o programa elege como os beneficiários exclusivos. Essas atividades definem também problemas focais que são as condições, deficiências ou carências para os quais as intervenções do PAB e incentivos variáveis estão direcionados.

As atividades do PSF são as que especialmente definem estratégias de acesso para as populações beneficiadas, como cadastros de famí- lias, desenvolvendo instrumentos de adscrição da população-alvo. Os dados de cobertura do PSF nos municípios definem a extensão em que o programa atinge a população-al vo.

$\mathrm{N}$ a segunda parte, trabalhamos com os primeiros resultados obtidos em função da alocação de recursos financeiros federais a favor de programas de Atenção Básica. Foi construída uma tipologia específica para a interpretação do impacto do programa PAB fixo sobre as contas municipais considerando a capacidade fiscal das unidades da Federação, seu porte populacional e região geográfica (Costa et al., 2002).

\section{A criação de novos programas}

As N ormas Operacionais Básicas de 1993 e 1996 (N O B 01/96) definiram uma série de processos automáticos de descentralização de recursos e instituíram uma política específica para a atenção básica, ao final favorecendo os programas considerados estratégi cos para o desenvolvimento de um novo modelo de assistência como o PAB Fixo, os de Saúde da Família (PSF) e os de Agentes Comunitários de Saúde (PACS), por meio de incentivos financeiros (portaria 1.886/GM , 18/12/1997).

A N OB/96 reforça o comando único do setor em nível local, amplia os mecanismos e fluxos de financiamento, reduzindo a remuneração contra a prestação de serviços e ampliando as transferências (fundo a fundo) com base na programação do uso dos recursos; a introdução de mecanismos de acompanhamento, controle e avaliação do SUS, valorizando os resultados advindos de programações com critérios epidemiológicos e desempenho de qualidade; e os vínculos dos serviços com seus usuários, privilegiando os núcleos familiares e comunitários e criando condições para uma maior participação e controle social. Cria dois níveis de gestão em nível municipal: a plena da atenção básica e a plena do sistema municipal. Nos dois casos existem pré-requisitos para que os municípios possam pedir adesão: na primeira situação, 0 município passa a receber integralmente os recursos destinados à atenção básica e tem autonomia para aplicá-los; e no segundo, a autonomia é estendida ao conjunto da assistência que envolve também procedimentos de média e alta complexidade hospitalar.

Durante 1997 foi lançado o programa 0 Ano da Saúdeno Brasil, estruturado em seismetas básicas: (a) a garantia de fontes estáveis de 
financiamento; (b) a descentralização dos serviços; (c) a reestruturação interna do M inistério da Saúde; (d) a fiscalização na aplicação dos recursos; (e) a prevenção com ênfase no atendimento básico; e (f) a melhoria da qualidade dos serviços. Também propunha a revisão da política de medicamentos básicos, o desenvolvimento dos programas de saúde da família e agentes comunitários de saúde, a redefinição da política de medicina supletiva, com a criação do Departamento de Saúde Suplementar e a realização dos primei ros estudos para a regulamentação dos mecanismos de seguro de saúde no Brasil. (M elamed, 2000)

Com relação a esses objetivos mais relacionados à questão do financiamento, foi feito, em resumo, um grande esforço para garantir fontes estáveis de financiamento, ou seja, a destinação exclusiva da CPM F e a aprovação da Proposta de Emenda Constitucional (PEC) 169. Procurou-se habilitar um grande número de estados e municípios para a gestão plena do sistema de saúde. Criaram-se incentivos especiais para programas já em curso como: (a) Programa de Agentes Comunitários de Saúde e de Saúde da Família; (b) Programa de Gestão do Atendimento Básico; (c) Programa da Farmácia Básica; (d) Combate a Grandes Endemias; Saúde da Mulher; e (e) Saúde da Criança. Foram estabelecidas prioridades em termos nacionais para a ampliação da rede de serviços públicos, através do projeto Reforsus, e foi feita uma revisão das tabelas do SUS, com base nas prioridades de saúde definidas pelo ministério, em comum acordo com representantes de estados e municípios, de forma a estimular o financiamento adequado dos procedimentos baseados em seu custo-efetivo.

Entre 1998 e 2000, além de serem mantidos os estímulos a todos os programas criados a partir da NOB/96, foi aprofundada a descentralização de recursos a partir de um conjunto de portarias que tiveram como objetivo descentralizar recursos, estabelecen do tetos para programas, entre os quais destacam-se o Programa de Atenção Básica ( $P A B$ ) e os programas incluídos sob a rubrica da Assistência Ambulatorial de M édia e Alta Complexidade. Os tetos para estados e municípios baseiam-se em média calculada na produção de serviços realizados nos seis meses anteriores. Há uma importante observação a fazer sobre as últimas medidas tomadas pelo M inistério da Saúde. Quando se calcula a média praticada por municípios em todo o país encontram-se resultados muito diferentes, re- fletindo a diversidade regional. Dessa forma, para que se possa alterar parcialmente as desigualdades constatadas sem reduzir o gasto em municípios que apresentam redes mais complexas, torna-se necessário buscar recursos "novos" para construir um patamar mínimo em todo 0 território nacional.

A partir de 1998, a rubrica para a Assistência Básica é separada da assistência médica em geral. 0 Piso de Assistência Básica foi criado pela portaria n. 84/GM e determina o valor máximo da parte fixa em $R \$ 18,00$ e o mínimo de $R \$ 10,00$, sempre baseado em média calculada sobre a produção do período anterior.

Os novos recursos aplicados diretamente em assistência são provenientes de uma reorganização interna das principais contas que compõem os balanços publicados pelo M inistério da Saúde - pessoal, assistência médica e dívida interna. Entre 1995 e 1999, constata-se que a média da participação do gasto total do ministério com assistência médica é de $55,54 \%$, enquanto a participação do item pessoal ativo e inativo fica em torno de $21,54 \%$. A alteração significativa para o período acontece em 1999, o total de custeio do MS (que exclui dívida e pessoal) alcançou seu maior percentual, $77,9 \%$, sendo também recorde em valores absolutos, como resultado da quitação quase integral da dívida interna do ministério que representou uma média de $10 \%$ do total de gastos entre 95 e 98 e caiu para menos de 1\% em 1999.

Contudo, as grandes modificações internas nas contas do ministério, perceptíveis apenas parcialmente em seus balanços, correm por conta da descentralização dos recursos que passam a ser manejados por uma grande parte de municípios, pelo mecanismo fundo (nacional) a fundo (municipal). Como é possível observar na tabela 1, as transferências para os municípios cresceram de $24,1 \%$ do total de recursos disponibilizados pelo M inistério da Saúde em 1997 para 66,90\% em 2001.

\section{A criação do Piso de Atenção Básica}

Há na literatura sobre financiamento à saúde no Brasil referências relativas à concentrada aplicação de recursos federais nas áreas mais ricas do país, que teoricamente acentuariam desigualdades ao invés de minorá-las (ver, por exemplo, M édici, 2001). N esses estudos, quase sempre toma-se como unidade de análise dados correspondentes às regiões geográficas e estados da Federação, sendo estes últimos pré-definidos 
Tabela 1

Indicadores de evolução da descentralização no SUS. Posição no final de cada exercício, 1997-2001

\begin{tabular}{|c|c|c|c|c|c|}
\hline Indicador/Ano & Dez/97 & Dez/98 & Dez/99 & Dez/00 & Dez/2001 \\
\hline N. de municípios recebendo recursos fundo a fundo & 144 & 5.049 & 5.350 & 5.450 & 5.516 \\
\hline N. de estados recebendo recursos fundo a fundo & - & 2 & 7 & 8 & 12 \\
\hline \% da população residente nos municípios que recebem recursos fundo a fundo & 17,3 & 89,9 & 99,26 & 99,72 & 99,90 \\
\hline$\%$ do total de recursos assistenciais transferidos fundo a fundo & 24,1 & 52,5 & 58,1 & 63,2 & 66,90 \\
\hline
\end{tabular}

Fonte: M inistério da Saúde - Dados de H abilitação /SAS/SPS (1997-2001), (M S, 2002)

como ricos e pobres. Trabalha-se com indicadores excessivamente abrangentes tais como: relação de profissionais de saúde e/ou número de leitos per capita, etc., na ausência de uma sintonia mais fina direcionada à apuração da oferta de serviços de saúde nos municípios, palco real da política de saúde brasileira dos anos 90 .

Outra área de fragilidade da bibliografia se relaciona a dispêndios per capita em saúde, somados às transferências federais. Afirma-se que estas apresentam baixo grau de correlação com o Produto Interno Bruto per capita de cada Estado, concluindo-se que o governo federal não tem orientado a distribuição das transferências aos estados baseando-se em critérios de eqüidade ou de necessidade de recursos ( $M$ édici, 2001).

Na maior parte das análises realizadas até 0 momento tem se ignorado, por exemplo, o peso do setor de saúde privado em cada região ou Estado, o grau de dependência da população do sistema SU S em seus diversos níveis de atenção. Qual a parcela dos serviços de saúde ofertados financiados por recursos próprios? Em que medida a capacidade fiscal municipal afeta o gasto em saúde? Como diferentes municípios se consorciam na oferta de diferentes serviços? E, por último, como a capacidade gerencial expressa pelo poder de atração de transferências mais volumosas vêem impactando a produção de serviços e os principais indicadores de saúde em cada município?

Durante a fase de estudos que antecedeu a criação do Piso de Atenção Básica, foi reforçada também a tese de que a distribuição dos dispêndios federais com procedimentos ambulatoriais, incluindo os de alta e média complexidade, acompanhava o quadro de concentração de renda e as desigual dades sociais.

Para rejeitar esses diagnósticos e para sustentar a hipótese do impacto distributivo dos novos programas de atenção ambulatorial, de- cidimos construir uma tipologia que considere o porte municipal, capacidade de financiamento baseada em uma proxy da arrecadação municipal e a dotação inicial a título de atenção básica como uma matriz padrão para a análise.

\section{Os municípios brasileiros e as transferências federais para a Atenção Básica}

É muito difícil a escolha de variáveis que possam ser utilizadas para a análise do desempenho do se tor público em saúde, adequadas ao universo de municípios no país, que permitam a formação de clusters eque, ao mesmo tempo, tenham a capacidade deexplicar o impacto redistributivo que exercem as tran sferências federais destinadas ao SUS sobre a produção de serviços e melhoria das condições de qualidadee acesso (Silva, 2000). Como já exposto, a partir de 1996, há um processo de descentralização em curso que tomou diferentes características em função da capacidade gerencial instalada em cada município e que permitiu diferentes níveis de habilitação. Ao final de 2000 a situação quese tinha está representada nos quadros $1 \mathrm{e} 2$.

É interessante observar que havia apenas 523 municípios habilitados em gestão plena desistema municipal, no entanto, congregando $38 \%$ da população enquanto que os 4.927 habilitados em Gestão Plena da Atenção Básica reúnem mais de $50 \%$.

Osíndices de correlação entre porte populacional, habilitação básica (IC $=81.35 \%)$ e plena do sistema (IC $=81.04 \%)$ são muito elevados. Essa situação sugere quea capacidade gerencial instalada em saúde esteja correlacionada com níveis de dimensão e concentração populacional, assim como se supõe que a produção ambulatorial ampliada, incluindo serviços de maior complexidade, mesmo a financiada apenas exclusivamente pelo setor 
Quadro 1

Número de municípios habilitados em gestão plena da Atenção Básica por região

\begin{tabular}{lcccc}
\hline Região & Número de municípios & População 2000 & Municípios habilitados & População coberta \\
\hline N orte & 449 & 12.919 .949 & 378 & 7.410 .799 \\
N ordeste & 1.792 & 47.679 .381 & 1.647 & 31.380 .466 \\
Centro-Oeste & 463 & 11.611 .491 & 420 & 5.738 .767 \\
Sudeste & 1.668 & 72.262 .411 & 1.369 & 24.418 .106 \\
Sul & 1.189 & 25.071 .211 & 1.113 & 17.162 .526 \\
Brasil & 5.561 & 169.544 .443 & 4.927 & 86.110 .664 \\
\hline
\end{tabular}

Fonte: Coord. Impl. Desc/ SPS - Gestão Plena da Atenção Básica (GPAB)

Quadro 2

N úmero de municípios habilitados em gestão plena do sistema municipal por região

\begin{tabular}{lcccc}
\hline Região & Número de municípios & População 2000 & Municípios habilitados & População coberta \\
\hline Norte & 449 & 12.919 .949 & 57 & 4.327 .569 \\
N ordeste & 1.792 & 47.679 .381 & 130 & 14.694 .167 \\
Centro-Oeste & 463 & 11.611 .491 & 24 & 3.507 .416 \\
Sudeste & 1.668 & 72.262 .411 & 269 & 34.955 .659 \\
Sul & 1.189 & 25.071 .211 & 43 & 7.276 .054 \\
Brasil & 5.561 & 169.544 .443 & 523 & 64.760 .865 \\
\hline
\end{tabular}

Fonte: Coord. Impl. Desc/ SPS - Gestão Plena da Atenção Básica (GPAB)

público funciona deacordo com economias deescala, devendo-se beneficiar da estrutura de serviços privados já existentes e credenciados ao SUS.

A hipótese levantada acima pôde ser confirmada quando se testou a correlação entre os recursos federais destinados ao conjunto da produção ambulatorial em 1998 e as receitas municipais em 1997 , o resultado éinferior a 30\% (IC=2.890). Isso significa que, ao menos, até 1998 havia municípios de médio e grande porte populacional que não re cebiam recursos suficientes e também não dispunham defontes próprias para financiar o conjunto das atividades relacionadas à produção ambulatorial proposta pela lei orgânica da saúde 8.080/90. Os dados indicam não haver uma relação explícita entre a capacidade de arrecadação fiscal municipal eo volume de produção ambulatorial, considerada em seu conjunto, envolvendo procedimentos de atenção básica, média ealta complexidade.

Por ocasião da definição de metodologia para a avaliação do impacto produzido pelo $\mathrm{PAB}$, desconsideramos inicialmente a forma de habilitação, etomamos como referência inicial a quantia que os municípios estavam recebendo a título de atenção básica em 1996, isto é, antes da instituição do novo piso pois supomos que os já habilitados como plena do sistema, em sua maior parte, por serem municípios de mais elevado porte populacional, não tiveram seus valores PAB significativamente alterados.

Obteve-se, então, cinco conjuntos distribuídos da forma como aparecem no quadro 3 eque passaremos deste ponto em diante neste relatório a denominar Grupos PAB.

Os mesmos grupos foram trabalhados segundo porte populacional, receita orçamentária, região do país, produção ambulatorial, eserviram de moldura para a análise dos principais resultados de processo eindicadores de resultado propostos pelo Pacto da Atenção Básica como passaremos a descrever a seguir.

\section{Análise dos Grupos PAB por características socioeconômicas}

No quadro 4, observa-seque aproximadamente $67 \%$ dos municípios brasileiros ou 3.261 municípios existentes à época (mais de 700 municípios foram criados no Brasil entre 1996 e 2000) receberam durante 1996 quantias inferiores a $R \$ 10,00$ 
Quadro 3

M unicípios brasileiros distribuídos por Grupos PAB - 1996

\begin{tabular}{lcr}
\hline Grupos PAB 96 & N. Municípios & \multicolumn{1}{c}{$\%$} \\
\hline Grupo 1 $(R \$ 0,0$ a $R \$ 3,0)$ & 787 & 16,23 \\
Grupo 2 $(R \$ 3,1$ a $R \$ 7,00$ & 1.399 & 28,86 \\
Grupo 3 $(R \$ 7,1$ a $R \$ 10,0)$ & 1.081 & 22,30 \\
Grupo 4 (R\$10,1 a $R \$ 20,0)$ & 1.436 & 29,62 \\
Grupo 5 (acima de $R \$ 20,0)$ & 145 & 2,99 \\
Total & 4.848 & 100,00
\end{tabular}

Fonte: Elaboração própria, dados primários $D$ atasus

per capita, a título do que convencionou-se chamar assistência básica eque a correlação mais evidenteéa de porte populacional, $90 \%$ desses municípios apresentavam até 50.000 habitantes, no total de 203 municípios com mais de 100.000 habitantes, $32 \%$ recebiam menos de $R \$ 7,00$ per capita em 1996.

Quanto à receita orçamentária (Quadro 5), aproximadamente $98 \%$ dos municípios dos grupos 1, 2 e 3 encontram-se atéo segundo extrato de arrecadação per capita escolhido para este trabaIho: no intervalo $R \$ 0,00$ a $R \$ 750,00$, este percentual cai ligeiramente para $95 \%$ no grupo 4 , indicando que a variável capacidade fiscal é muito fra ca para explicar flutuações em aplicações financeiras realizadas pelos municípios brasileiros em saúde.

Os mesmos grupos PAB analisados de acordo com as regiões do país ( $Q$ uadro 6) indicam que $65 \%$ dos municípios do grupo 1 estavam na região Norte ou Nordeste. Essa proporção cai para 39\% no grupo 2. Os municípios das regiões Sudestee Sul correspondem respectivamente às proporções de $54 \%$ e $63 \%$ no interior dos grupos 3 e 4 . Apesar desses percentuais demonstrarem uma relativa concentração dos municípios da região Norte entre os gruposque recebiam valores inferioresà mé dia nacional, a título de assistência básica, antes da instituição do PAB edeos municípios do Sul eSudeste estarem majoritariamente representados entre os grupos 3 e 4, a distribuição de municípios nordestinos é bastante equilibrada entre os quatro grupos PAB. M ais uma vez, fica reforçada nossa hipótese original de quehá municípios com características similares para o conjunto de variáveis que trabalhamos até aqui, espalhados por todas as regiões do país. É importante destacar, contudo, que os municípios dos grupos 1 e 2 foram os principais beneficiários das mudanças nos mecanismos de transferência propostos pelo PAB. N esse sentido, é defensável a hipótese do impacto redistribu- tivo da política de saúde quando tomada como variável de análise o conjunto dos municípios brasileiros.

\section{Avaliação das inovações no financiamento}

O novo mecanismo de distribuição dos recursos inaugurado pela N OB/96 visou paulatinamente dissociar o faturamento dos serviços de saúde de seu financiamento. Na situação anterior, municípios demenor porte e/ ou mais pobres não prestavam assistência básica à saúde, por não disporem de capacidade instalada e/ou recursos próprios para produzirem serviços na proporção das necessidades da população local.

A implantação do PAB traz como principal mecanismo a transferência regular eautomática, 0 que se traduz em um repasse federal feito diretamente aos municípios. Esse novo mecanismo dissocia a produção do faturamento, característica central do sistema anterior. Isto é, de acordo com a lógica de pagamento por serviços previamente prestados, os municípios mais pobres e/ou de me nor porte, portanto, sem uma infra-estrutura adequada de unidades e de estabelecimentos de saúde, acabavam prejudicados. Comparados aos municípios mais populosos, recebiam do SUS valores me nores, porém coerentes, por um lado, com a precariedade da rede de saúde existente no local, e por outro, com a quantidade de procedimentos eatendimentos realizados. Ainda, de acordo com o critério anterior, quanto maior o número de hospitais, ambulatórios e postos de saúde, maior a possibilidade do município receber aportes financeiros junto ao SUS. Além disso, o antigo sistema, baseado no faturamento de consultas e de procedimentos real izados, perpetuava um modelo assistencial com pouca ou nenhuma ênfase na adoção de medidas preventivas e/ou de promoção à saúde eredução de agravos.

Em síntese, toda a transformação proposta pe lo conjunto de orientações nacionais para a política deatenção básica à saúdena década de 1990 podeser resumida em quatro grandes objetivos: 1) alterar a forma de al ocação de recursos federais destinados à produção de serviços básicos de saúde, visando criar estímulos à co-participação de estados e municípios, à ampliação da capacidade instalada e emprego de recursos humanos, particularmenteem municípios mais carentes; 2) criar estímulos específicos para reorientar a estrutura de oferta de serviços básicos visando a uma maior homogeneidadeem nível nacional, com ên- 
Quadro 4

M unicípios brasileiros por Grupos-PAB (1996) , porte populacional

\begin{tabular}{lrrrrr}
\hline Grupos PAB 96 & G1 & G2 & G3 & G4 & G5 \\
\hline Port1 até 10.000 hab & 322 & 599 & 409 & 669 & 103 \\
Port2 10.001 a 50.000 hab & 416 & 670 & 519 & 605 & 39 \\
Port3 50.001 a 100.000 hab & 30 & 78 & 79 & 99 & 2 \\
Prot4 acima de 100.000 hab & 13 & 52 & 74 & 63 & 1 \\
Total & 781 & 1.399 & 1.081 & 1.436 & 145 \\
\hline
\end{tabular}

Fonte: Elaboração própria, dados primários - STN -M F e IBGE

Quadro 5

Municípios brasileiros por Grupos-PAB (1996), por Receita Orçamentária per capita

\begin{tabular}{lrrrrr}
\hline Grupos PAB 96 & G1 & G2 & G3 & G4 & G5 \\
\hline R01/Cap - R $\$ 0,00$ a $R \$ 250,00$ & 498 & 730 & 513 & 527 & 37 \\
R02/Cap - R $\$ 250,01$ a $R \$ 750,00$ & 272 & 639 & 540 & 839 & 101 \\
R03/Cap - acima de $\$ \$ 750,01$ & 11 & 30 & 28 & 70 & 7 \\
Total & 781 & 1.399 & 1.081 & 1.436 & 145 \\
\hline
\end{tabular}

Fonte: Elaboração própria, dados primários - STN-M F e IBGE

Quadro 6

Municípios - Grupo PAB 1996 por região

\begin{tabular}{lcccccr}
\hline Grupos PAB 96 & Grupo 1 & Grupo 2 & Grupo 3 & Grupo 4 & Grupo 5 & Total \\
\hline Região 1 & 155 & 98 & 56 & 51 & 10 & 370 \\
Região 2 & 354 & 448 & 337 & 357 & 44 & 1.540 \\
Região 3 & 50 & 307 & 459 & 618 & 45 & 1.479 \\
Região 4 & 170 & 409 & 133 & 292 & 34 & 1.038 \\
Região 5 & 58 & 137 & 96 & 118 & 12 & 421 \\
Total & 787 & 1.399 & 1.081 & 1.436 & 145 & 4.848 \\
\hline
\end{tabular}

Fonte: Elaboração própria, dados primários - STN -M F e IBGE

fase na proliferação de ações e procedimentos de atenção básica e de medidas de promoção e prevenção em saúde;

3) construir um sistema de informações estruturado com base nas informações fornecidas diretamentepor gestores municipais objetivando a constituição de um sistema nacional de maior qualidade eque permitam uma avaliação segura dos novos programas; e

4) promover mudanças nos indicadores de saúde para o conjunto do país que comprovem a efetividade específica dos novos programas sob a gestão direta dos municípios e supervisão dos estados.

0 programa PAB foi muito bem-sucedido, se considerada a eficiência demonstrada pelos municípios na habilitação às novas condições de gestão exigidas, ena presteza do governo federal em alocar os recursos necessários para a efetivação da nova política. A partir de 1998, chega-se ao total de $\mathrm{R} \$ 1.174$ bilhões distribuídos a título de PAB para todos os municípios do país. Em 1999 chega-sea um valor total em torno de $\mathrm{R} \$ 1.700$ bilhões, que se manteve constantenos anos posteriores. Foram atingidas médias semelhantes quer se considerea 
distribuição de recursos por regiões ou por grupos $\mathrm{PAB}$, constituindo-se em uma política redistributiva sem precedentes no caso brasileiro.

No caso dos Programas de Agentes Comunitá rios (PACS) e Saúde da Família (PSF), a dinâmica de incorporação dos repasses financeiros por parte dos municípios se deu de forma um pouco mais lenta no tempo por implicar contrapartida de dispêndios municipais, como é possível observar nos quadros 7 e 8. Em 1998, o PACS já estava bem espalhado pelo Nordeste, mas atingia apenas 3.291 municípios. Em 2001, esse número cresce para 4.858, contudo, encontrando-se mais concentradamente incorporado nas regiões $\mathrm{N}$ orte e $\mathrm{N}$ ordeste do que Sul eCentro-oeste, especialmente pelo volume de recursos aplicados; apenas 19\% dos recursos totais são destinados a estas duas últimas regiões, enquanto o Sudeste recebeu $20 \%$.

O Programa de Saúde da Família, ainda de incorporação mais lenta, apresentou uma inserção mais do que triplicada ao longo dos últimos quatro anos, atingindo 1.218 municípios em 1998 e cerca de 3.796 em 2001, tendo sido para esteúlti- mo ano $45 \%$ do recursos disponibilizados para o Nordeste

\section{Conclusões}

Este artigo demonstra que o processo de descentralização da atenção básica à saú de no Brasil definiu uma estrutura de regras e de incentivos positivos que favorecem a transferência direta de recursos federais para a esfera municipal, tendo os gestores de saúde locais ganho el evado poder decisório sobre recursos que passaram a ser manejados por meio de mecanismos fundo (nacional) a fundo (municipal).

Essas evidências sugerem que sejam revistas as teses preval entes na literatura sobre financiamento à saúde no Brasil que se sustentam sobrea idéia da persistente concentração das transferências de recursos federais nas áreas mais ricas do país, que teoricamente acentuariam desigual dades ao invés de minorá-las. Na literatura, tomouse como unidade de análise os dados para as re-

Quadro 7

Programa Agente Comunitário de Saúde - PACS

\begin{tabular}{lrrrrrrrr}
\hline Região & \multicolumn{2}{c}{1998} & \multicolumn{2}{c}{1999} & \multicolumn{2}{c}{$\mathbf{2 0 0 0}$} & \multicolumn{2}{c}{$\mathbf{2 0 0 1}$} \\
& N. municípios & \multicolumn{1}{c}{ Valor R\$ } & N. municípios & \multicolumn{1}{c}{ Valor R\$ } & N. municípios & Valor R\$ & N. municípios Valor R\$ \\
\hline 1 & 310 & $13.060 .775,25$ & 391 & $29.966 .017,21$ & 424 & $43.307 .511,27$ & 437 & $42.871 .227,78$ \\
2 & 1.421 & $59.211 .741,67$ & 1.723 & $119.085 .015,14$ & 1.766 & $144.909 .206,54$ & 1.784 & $140.574 .926,55$ \\
3 & 707 & $9.844 .492,13$ & 815 & $18.983 .662,85$ & 1.072 & $42.543 .655,55$ & 1.221 & $61.058 .638,99$ \\
4 & 579 & $7.929 .950,11$ & 722 & $17.812 .301,26$ & 898 & $31.634 .762,15$ & 993 & $34.631 .476,03$ \\
5 & 274 & $8.249 .274,82$ & 306 & $14.966 .049,98$ & 359 & $20.705 .595,10$ & 423 & $23.227 .211,63$ \\
Total & 3.291 & $98.296 .233,98$ & 3.957 & $200.813 .046,44$ & 4.519 & $283.100 .730,61$ & 4.858 & $302.363 .480,98$ \\
\hline
\end{tabular}

Fonte: Elaboração própria, dados primários - Datasus-MS

\section{Quadro 8}

Programa Saúde da Família - PSF

\begin{tabular}{lrrrrrrrr}
\hline Região & \multicolumn{2}{c}{1998} & \multicolumn{2}{c}{1999} & \multicolumn{2}{c}{$\mathbf{2 0 0 0}$} & \multicolumn{2}{c}{$\mathbf{2 0 0 1}$} \\
& N. municípios & \multicolumn{1}{c}{ Valor R\$ } & N. municípios & \multicolumn{1}{c}{ Valor R\$ } & N. municípios & Valor R\$ & N. municípios Valor R\$ \\
\hline 1 & 103 & $1.667 .166,01$ & 216 & $8.543 .807,13$ & 278 & $26.512 .399,00$ & 309 & $34.357 .299,00$ \\
2 & 406 & $17.001 .415,21$ & 703 & $46.370 .175,91$ & 1.067 & $160.488 .741,54$ & 1.307 & $246.258 .697,00$ \\
3 & 535 & $16.785 .328,61$ & 631 & $33.531 .616,19$ & 916 & $104.502 .010,99$ & 1.106 & $147.498 .991,75$ \\
4 & 142 & $5.288 .081,73$ & 204 & $11.472 .271,44$ & 489 & $52.179 .245,00$ & 690 & $77.227 .452,50$ \\
5 & 32 & $4.724 .832,88$ & 69 & $5.859 .592,39$ & 245 & $28.260 .202,00$ & 384 & $49.860 .552,27$ \\
Total & 1.218 & $45.466 .824,44$ & 1.823 & $105.777 .463,06$ & 2.995 & $371.942 .598,53$ & 3.796 & $555.202 .992,52$ \\
\hline
\end{tabular}

Fonte: Elaboração própria, dados primários - Datasus-M S 
giões geográficas e estados da Federação, sendo estes últimos pré-definidos como ricos e pobres. Trabalha-se com indicadores excessivamente abrangentes tais como: relação de profissionais de saúde e/ou número de leitos per capita, etc., que não ajudam a discriminar o real impacto de programas específicos.

A análise dos dados de implementação do PAB entre 1998-2001 demonstra que os municípios de menor capacidade instalada para a oferta de atenção ambulatorial básica no âmbito do Sistema Ú nico de Saúde foram os mais favorecidos pel os novos mecanismos de transferência propostos pe las N ormas O peracionais Básicas (NOBs).

As N ormas O peracionais Básicas de 1993 e 1996 (N OB 01/96) foram bem-sucedidas na implementação de processos automáticos de descentralização de recursos e instituição de uma política específica para a atenção básica que favorecem os programas considerados estratégicos para o desenvolvimento de um novo modelo de assistência como os de saúde da família (PSF) e os de agentes comunitários de saúde (PACS), por meio de incentivos financeiros.

A implementação do PAB fixo teve elevado impacto na equalização das transferências federais para a atenção ambulatorial.

\section{Referências bibliográficas}

Costa et al. 2002. Relatório Final do Projeto Custo eAvaliação do Impacto da Implantação da Parte Fixa do Piso de Atenção Básica - PAB. M inistério da Saúde/ReforSUS/FENSPTEC.

M édici A 2001. Financiamiento y gasto público en salud en los años noventa. División de Programas Sociales 1 del Banco Interamericano de Desarrollo, (BR-009).

M elamed C 2000. Estrutura tributária, gasto social e financiamento da saúde pública no Brasil - anos 90. Tese de doutorado do IE da UFRJ.

M inistério da Saúde 2002. Reduzindo as desigualdades e ampliando 0 acesso à assistência à saúde no Brasil (1998-2002). Brasília.
Essas conclusões podem sugerir uma mudança no foco da análise da política de saúde para a atenção ambulatorial básica, na qual estão também localizados o PACS e PSF, para um enfoque orientado para a análise da sustentabilidade do conjunto desses programas e da qualidade dos serviços de saúde dirigidos a atenção básica.

É necessário incentivar novos estudos para estimar impacto das mudanças na forma de alocação de recursos federais destinados à produção de serviços básicos de saúde sobre a efetiva condição de co-participação de estados e municípios na ampliação de capacidade instalada e emprego de recursos humanos, particularmente dos municípios mais carentes. Essa questão torna-se ainda mais fundamental se considerada a questão da referência aos níveis de maior complexidade na atenção à saúde.

Cabe também responder se os estímulos específicos para reorientar a estrutura de oferta de serviços básicos, defato, alteram o modo de produção de atenção médica viabilizando maior ênfase de ações de promoção da saúde e redução de agravos e, ainda, se todas as novas orientações levarão a mudanças sensíveis nos indicadores de saúde.
Piola SF \& Biasoto Jr G 2001. Financiamento do SUS nos anos 90, pp. 102-110. In B N egri \& G Giovanni (orgs.). Brasil: radiografia da saúde. Unicamp, Campinas.

Piola SF \& Vianna SM 1999. Descentralização e gestão do gasto público com saúde no Brasil, pp. 105-140. In Compêndio de documentos do CEPAL/BID/I pea: Taller sobre evaluación de la gestión del gasto publico en salud. Cepal, Brasília.

Silva PB et al. 2000. Relatório Final do Projeto Avaliação da Implementação do PAB. NEPP-Unicamp, Campinas.

Artigo apresentado em 20/10/2002

Aprovado em 14/1/2003

Versão final apresentada em em 6/6/2003 